\title{
DIMENSIONES DE LA CAPACIDAD DE ABSORCIÓN DEL CONOCIMIENTO Y LA VINCULACIÓN DEL POSGRADO EN INGENIERÍA INDUSTRIAL DE LA UNIVERSIDAD DE SONORA
}

\author{
Dimensions of the absorptive capacity of knowledge and the linkage of the \\ postgraduate in industrial engineering of the university of sonora
}

EPISTEMUS

ISSN: 2007-8196 (electrónico)

ISSN: 2007-4530 (impresa)

Alonso Perez-Soltero 1
Leslie García Montijo

Recibido: 19 de septiembre de 2017

Aceptado: 20 de enero de 2018

Autor de Correspondencia:

Dr. Alonso Perez-Soltero

Correo: aperez@industrial.uson.mx

\section{Resumen}

En el presente artículo se realiza un análisis enfocado en la percepción de la industria sobre los proyectos de colaboración establecidos con el Posgrado en Ingeniería Industrial de la Universidad de Sonora con el objetivo de conocer la influencia de la Capacidad de Absorción del Conocimiento hacia la vinculación; así como las fortalezas y debilidades de cada una de sus dimensiones: Adquisición, Asimilación, Transformación y Explotación de conocimiento; haciendo énfasis en la aplicación del conocimiento y la tecnología por parte de los estudiantes del Posgrado para resolver problemas que afectan la calidad y productividad de las organizaciones en estudio; impulsando la mejora y fortalecimiento de procedimientos $y$ políticas internas para los proyectos de vinculación futuros.

Palabras clave: Capacidad de Absorción del Conocimiento, Vinculación.

\begin{abstract}
Abstract. In the present article performed an analysis focused on the perception of the industry on the collaboration projects established with the Postgraduate in Industrial Engineering of the University of Sonora with the objective of knowing the influence of the Absorptive Capacity of Knowledge towards the bonding; as well as the strengths and weaknesses of each of its dimensions: Acquisition, Assimilation, Transformation and Exploitation of knowledge; emphasizing the application of knowledge and technology by students of the Postgraduate to solve problems that affect the quality and productivity of the organizations under study; promoting the improvement and strengthening of internal procedures and policies for future linkage projects.
\end{abstract}

Keywords: Absorptive Capacity of Knowledge, Linkage.

1 Universidad de Sonora, México / Correo: aperez@industrial.uson.mx 


\section{INTRODUCCIÓN}

En el presente estudio se realiza un análisis de la situación actual de la capacidad de absorción del conocimiento en empresas que han participado en proyectos de colaboración con el Posgrado en Ingeniería Industrial de la Universidad de Sonora. Se identifican las debilidades y fortalezas por dimensión: Adquisición, Asimilación, Transformación y Explotación. Este trabajo estuvo dirigido a los empresarios que estuvieron trabajando directamente en proyectos de colaboración con estudiantes del Posgrado.

El objetivo principal de este estudio es analizar la capacidad de absorción del conocimiento por medio de la percepción de las empresas participantes en los proyectos de colaboración con el Posgrado en Ingeniería Industrial para fortalecer los procedimientos y políticas de vinculación existentes. Estas experiencias servirán para retroalimentación de las prácticas actuales de vinculación; así como la definición, comportamiento, desarrollo y resultado de dichas colaboraciones con la finalidad de mejorar procesos y políticas de vinculación. La relevancia de la Capacidad de Absorción del Conocimiento y el comportamiento de cada una de las dimensiones se presenta en un diagnóstico de fortalezas y debilidades percibidas por parte de los empresarios involucrados directamente; así como el panorama actual y el deseado por dimensión referente a la vinculación y el nivel de satisfacción obtenido por los proyectos de colaboración.

La estructura del trabajo inicia describiendo la importancia de la capacidad de absorción del conocimiento y sus dimensiones, luego se describe el método del estudio, posteriormente los resultados obtenidos y finalmente las conclusiones.

\section{IMPORTANCIA DE LA CAPACIDAD DE ABSORCIÓN DEL CONOCIMIENTO Y SUS DIMENSIONES.}

La capacidad de absorción de conocimiento es un concepto que se comprende como la habilidad

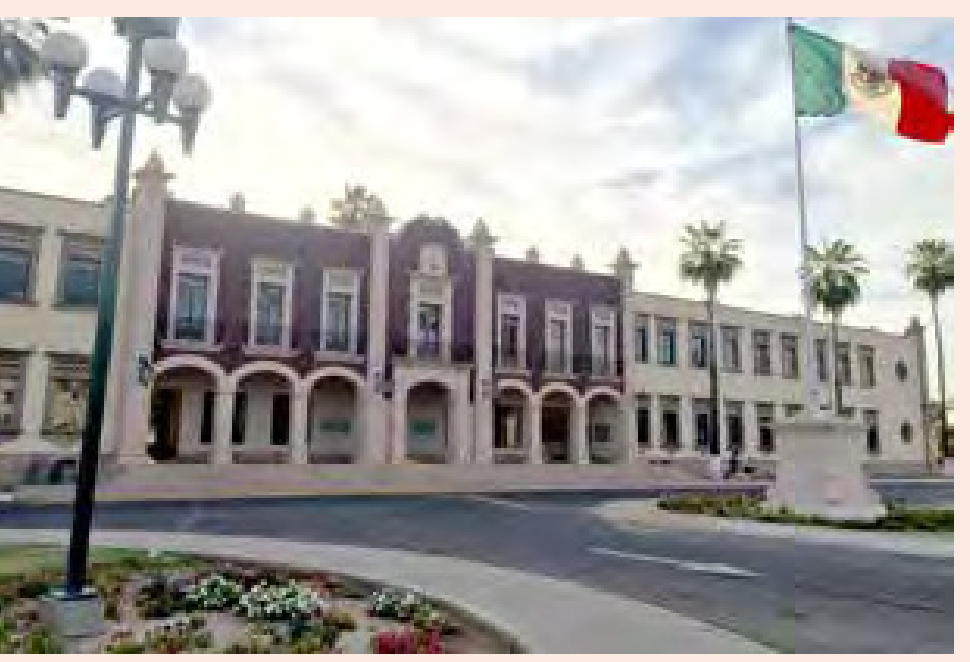

organizacional para identificar, asimilar, transformar y explotar el conocimiento externo. A su vez, este concepto señala no solo la importancia del conocimiento externo si no la captación del conocimiento interno para interactuar dentro de una rutina organizacional mediante mecanismos que promuevan las interacciones externas y permitan adquirir el conocimiento disponible y adaptarlo de forma conveniente a la organización. La capacidad de absorción es un conjunto de habilidades claves que interactúan para expresarse con el propósito de innovar, varios autores la definen como se observa en la Tabla 1.

\section{Tabla 1. Definiciones de Capacidad de Absorción del Conocimiento.}

\footnotetext{
Definición de Capacidad de

Absorción del Conocimiento

"Habilidad para reconocer el valor de nueva información externa, asimilarla, y aplicarla a fines comerciales."

"Las empresas reconocen el valor, adquieren, transforman o asimilan y explotan el conocimiento."

"Habilidad de una empresa para utilizar el conocimiento obtenido del exterior a través de tres procesos secuenciales y obtener resultados comerciales."
}

Según:

"Habilidad relativa de una empresa estudiante para valorar, asimilar y aplicar el nuevo conocimiento de una empresa maestra."

"Conjunto de rutinas
organizacionales y procesos
estratégicos a través de los
cuales las empresas adquieren,
asimilan, transforman y explotan
conocimiento con el propósito de
crear valor."

Lane y Lubatkin [4] Lane, Koka y Pathak [3]

Cohen y Levithal [1] Todorova \& Durisin [2] 3]

\section{DEFINIENDO LAS DIMENSIONES DE LA CAPACIDAD DE ABSORCIÓN DEL CONOCIMIENTO}

Adquisición. Es la capacidad de la empresa para identificar, valorar, seleccionar y adquirir conocimiento externo [5] crítico para sus operaciones con fines comerciales [4].

Asimilación. Está definida como la capacidad de la empresa para analizar, procesar, interpretar, internalizar y clasificar el nuevo conocimiento externo[3] adquirido [6].

Transformación. Su principal objetivo es establecer cómo adaptar el nuevo conocimiento a la realidad y necesidades de la organización [5]. La capacidad de transformación puede ser alcanzada mediante la adición o eliminación de conocimiento existente, o mediante la 
combinación del conocimiento de formas novedosas y radicalmente diferentes [7].

Explotación. Es la utilización del nuevo conocimiento absorbido con fines comerciales [4]. Aplicándolo en rutinas, operaciones, procesos con la finalidad de mejorar o desarrollar el conocimiento existente, creando nuevas prácticas y capacidades en la empresa [5].

\section{MÉTODO}

Este estudio se enfoca en conocer la percepción de los empresarios involucrados directamente en los proyectos de colaboración con los estudiantes del Posgrado en Ingeniería Industrial. La finalidad es la de conocer, cual es la capacidad de absorción del conocimiento por parte de la empresa y cómo se ve influenciada la vinculación.

Primeramente se creó y validó el instrumento de medición mediante Alfa de Cronbach (0.867) para medir su grado de confiabilidad [8]. Posteriormente se aplicó el instrumento a las empresas involucradas; de las respuestas obtenidas, se realizó un análisis de fortalezas y debilidades por dimensiones: Adquisición, Asimilación, Transformación y Explotación del conocimiento; así como el grado de Satisfacción con la relación a la vinculación.

Para este estudio, se contó con la información de los proyectos realizados entre el 2011 al 2016. Hasta el momento, 76 proyectos de vinculación entre el Posgrado en Ingeniería Industrial de la Universidad de Sonora y la Industria se han concluido. El instrumento va dirigido a los empresarios responsables que participaron en estos proyectos. De los 76 responsables por parte de la industria se localizaron a 40 de ellos.

En base a la fórmula para poblaciones finitas, se obtiene una muestra de 28 encuestas a aplicar obteniendo una respuesta de 22 empresarios que fueron responsables de proyectos de colaboración.

\section{RESULTADOS}

A continuación se muestran los resultados del estudio en cuanto a las dimensiones de la capacidad de absorción del conocimiento: adquisición, asimilación, transformación y explotación y su comportamiento en los proyectos de colaboración con la industria. A su vez, se presenta el panorama de la situación actual por dimensión y la situación deseada para la vinculación academia-industria.

\section{Adquisición del conocimiento y la vinculación}

El objetivo de esta sección fue identificar; cómo se formalizó la vinculación entre Academia-Industria. Un $90 \%$ de los empresarios contestaron que los proyectos de vinculación se dieron gracias al acercamiento de los estudiantes del Posgrado. Esta situación podría presentarse por varios factores: falta de información o un medio de enlace con el sector productivo que alerte del recurso disponible para efectuar proyectos de colaboración; falta de interés por parte de las empresas y/o en este caso el beneficio que el estudiante obtiene (grado de Maestro) por la vinculación, ya que es el motivo más fuerte para que el estudiante realice el enlace apresurando el encuentro con el empresario para concretar un compromiso de colaboración. El $80 \%$ de los empresarios participantes en proyectos de vinculación dijeron que tenían conocimiento de la existencia de los proyectos de vinculación que ofrecía el Posgrado y un $20 \%$ contestaron que No.

El $65 \%$ de los proyectos de colaboración por los cuales la empresa adquirió este conocimiento fueron enfocados para mejorar procesos y otro $25 \%$ para mejoras en Gestión Empresarial.

Basándose en el concepto de innovación abierta y en la forma de desarrollo e implementación del proyecto, se pregunta al responsable del proyecto en la empresa si se informó al resto de la organización que el estudiante del Posgrado estaría desarrollando un proyecto de colaboración. Un $55 \%$ de los responsables de proyecto contestó que les comunicó de la presencia del estudiante a todas las partes involucradas de donde pudiese necesitar información, y un 35\% solo informó al departamento donde se realizaría el proyecto.

En cuanto a la etapa de desarrollo del proyecto se requiere mayor compromiso por parte del Director de Tesis para apoyo y respaldo hacia el estudiante dentro de la empresa. Los responsables de la empresa señalaron que en un $50 \%$ de los proyectos solamente participó directamente el estudiante y representante de la empresa; y bajo una participación moderada el director de tesis del proyecto. El 90\% de los estudiantes se vincularon para el desarrollo de un proyecto especial y el $10 \%$ restante para integrarse a un equipo que ya trabajaba en un proyecto en desarrollo. A continuación en la Tabla 2 , se resumen los hallazgos obtenidos; en cuanto a fortalezas y debilidades en esta etapa del instrumento:

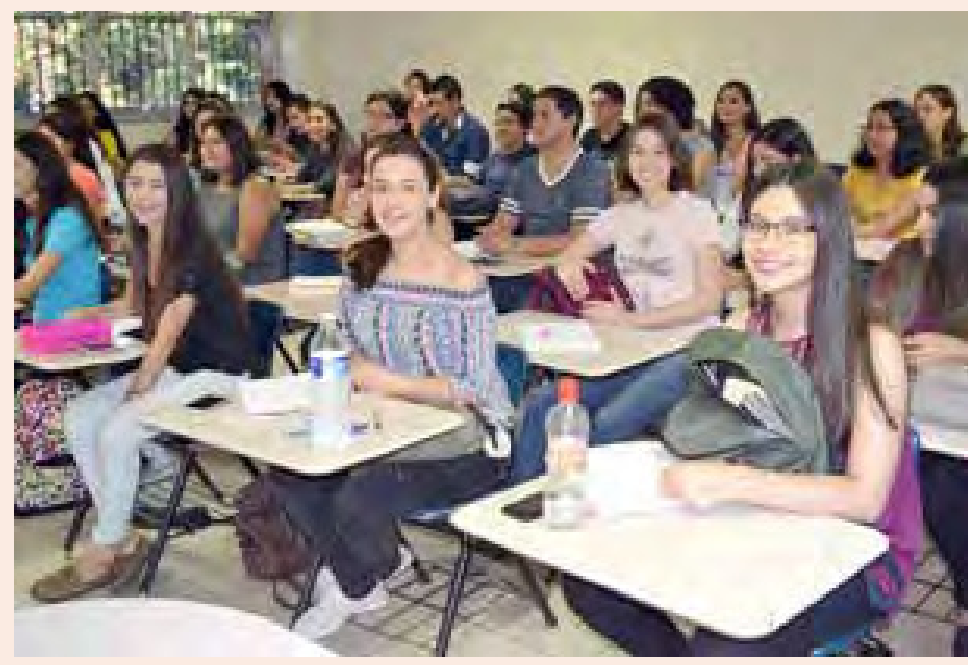


Tabla 2. Fortalezas y debilidades de la Adquisición.

\section{Fortalezas}

Interés por parte del estudiante del Posgrado para concretar vinculación academia-industria.

Todos los proyectos de vinculación participantes han generado innovación; ya sea en mejoras en procesos o mejoras en gestión empresarial.

Las empresas están abiertas ante la posibilidad de adquirir conocimiento siempre y cuando se acuda a ellas directamente por parte de la academia.

\section{Debilidades}

Falta de interés por parte de las empresas en acudir a la academia para concretar vinculación.

No se comunica por parte de la empresa a todos los departamentos involucrados que se realizará un proyecto en colaboración con la academia. Lo cual podría reducir o complicar la información proporcionada al estudiante.

Los participantes del equipo de trabajo en la investigación del proyecto no son constantes o no cumplen con las funciones asignadas.

\section{Panorama de la situación Actual y Deseada de la Dimensión Adquisición}

En cuanto a la situación deseada de la Dimensión Adquisición (Gráfico 1) las empresas conocen la existencia de los programas de vinculación que ofrece el Posgrado; no obstante, los mecanismos de vinculación para adquirir conocimiento, están alejados de la situación deseada, donde el acercamiento en este caso de estudio es por parte de la academia. La empresa no presenta la iniciativa de formalizar el vínculo de adquisición del conocimiento desaprovechando el recurso existente que le brinda la academia. El involucramiento por parte del empresario responsable que adquiere el conocimiento es de grado medio al momento de insertar al estudiante en la organización. La empresa se encuentra actualmente en la posición de retribuir por la adquisición del conocimiento futuro en base a la experiencia de colaboración previa con el Posgrado.

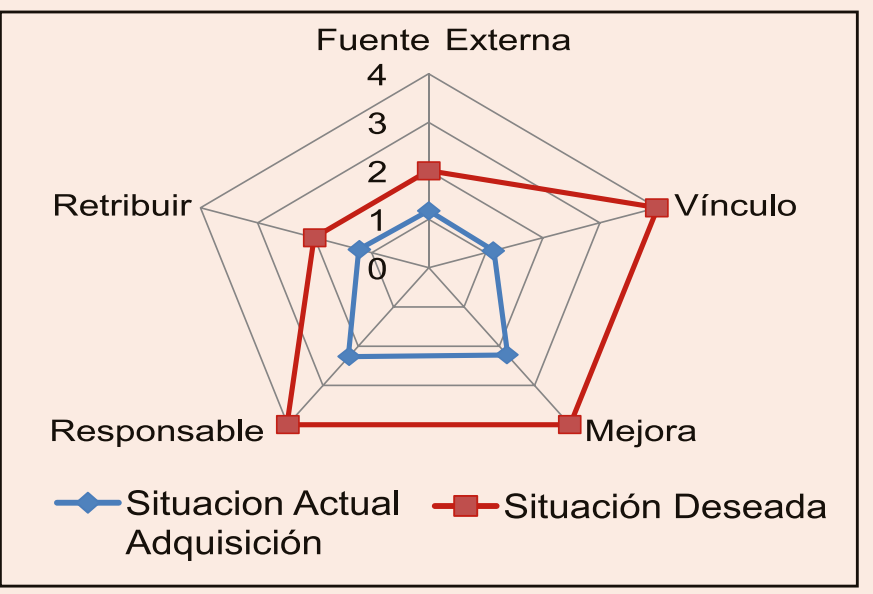

Gráfico 1. Situación Actual vs Deseada Adquisición

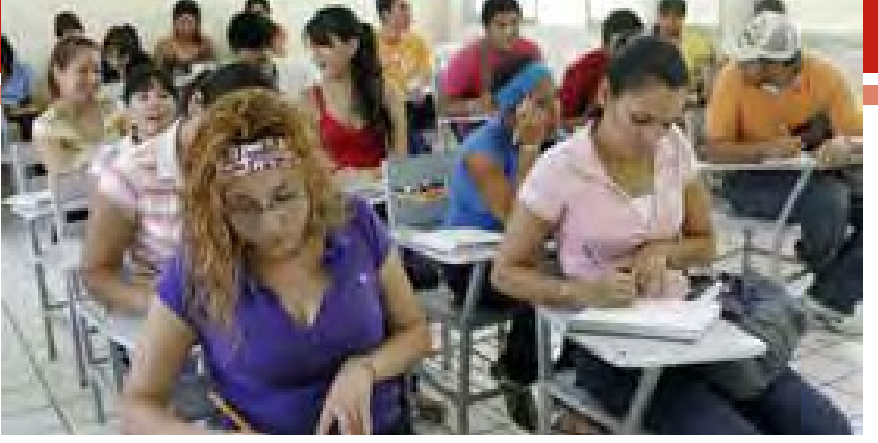

\section{Asimilación del conocimiento y la Vinculación.}

El objetivo de esta sección fue el de obtener información sobre las circunstancias en las cuales se desarrolló el proyecto. El proyecto de vinculación se desarrolla durante el tiempo de duración del programa de estudio (dos años). Para conocer la participación de los miembros de desarrollo de proyecto, se le preguntó al responsable por parte de la empresa cuál fue la frecuencia con la que acudieron los responsables por parte del Posgrado. Durante todo el desarrollo del proyecto, un $55 \%$ de los responsables de la empresa mencionaron que el Director de Tesis nunca se presentó; y referente a la asistencia del estudiante, el 50\% contestó que era de una o dos veces al mes. En cuanto a la etapa de desarrollo del proyecto, señalaron que en un $50 \%$ de los proyectos solamente participó directamente el estudiante y representante de la empresa; y bajo una participación moderada el Director de Tesis del proyecto. En cuanto a la etapa de implementación el $80 \%$ de los estudiantes captó totalmente las necesidades de la empresa y un $15 \%$ medianamente. Este aspecto se verá directamente reflejado en los resultados de la siguiente etapa que es la satisfacción con la relación de vinculación.

Tabla 3. Fortalezas y debilidades de la Asimilación.

Fortalezas $\quad$ Debilidades

El estudiante capta las necesidades y problemática de la empresa casi siempre en su totalidad.

No hay involucramiento por parte del Director de Tesis del proyecto en la empresa, lo cual podría debilitar la relación, formalidad y compromiso con la empresa.

El estudiante es capaz de liderar el proyecto con o sin el apoyo del Director de Tesis.

La frecuencia y horarios deberán ser establecidos por la empresa, no existe continuidad o lineamiento que lo estipule.

\section{Panorama de la situación Actual y Deseada de la} Dimensión Asimilación

En cuanto a la situación deseada de la Dimensión Asimilación (Gráfico 2) la academia deberá poner mayor énfasis en el apoyo académico (soporte y presentación del estudiante) los conocimientos específicos presentados por los estudiantes hacia la solución de los problemas de las empresas son de nivel medio; pero al momento de realizar el análisis y la investigación para la asimilación de las necesidades de las empresas el nivel es casi el deseado. 
Mejorando el nivel de involucramiento, es probable se cumpla con la situación deseada por parte de la empresa.

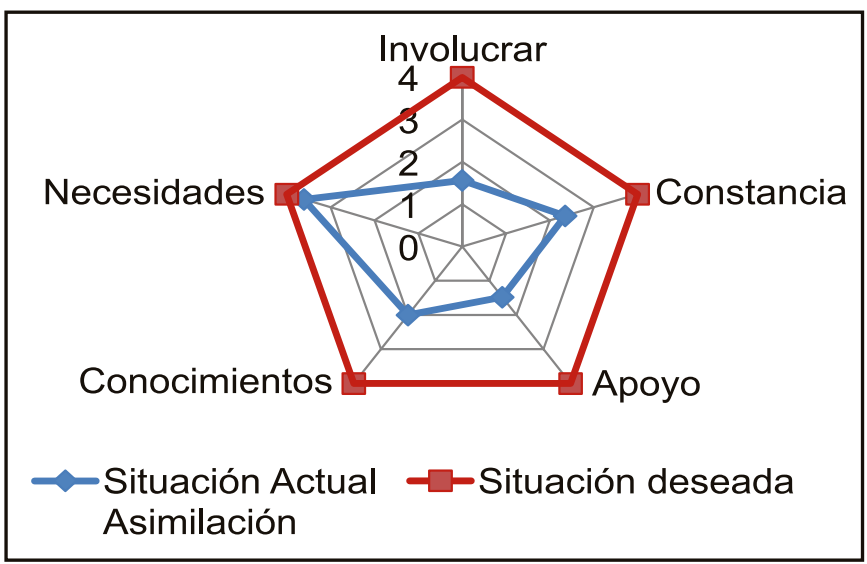

Gráfico 2. Situación Actual vs Deseada Asimilación

\section{Transformación y Explotación del conocimiento y la vinculación}

Referente a la Dimensión Transformación/Explotación en los proyectos de vinculación, la empresas calificaron en un 45\% como de "Alto" el impacto positivo generado por los proyectos de colaboración con estudiantes del Posgrado un $40 \%$ dice que impactó Medianamente. El $85 \%$ de las empresas señala que el impacto fue positivo y de total o mediana aceptación. Los resultados de los proyectos de vinculación fueron de innovación en procesos y productos en un $65 \%$ y en mejora de Gestión empresarial en un $25 \%$. El $55 \%$ de los proyectos han sido implementados total o parcialmente.

\section{Tabla 4. Fortalezas y Debilidades de la Transformación/ Explotación.}

\section{Fortalezas}

La mayoría de las empresas que participaron en los proyectos de vinculación manifestaron un impacto positivo en cuanto a la colaboración con los estudiantes.

Más de la mitad de los proyectos de vinculación han sido implementados

Los empresarios involucrados están dispuestos a participar en proyectos futuros con el Posgrado
Panorama de la situación Actual y Deseada de la Dimensión Transformación/ Explotación en los proyectos de vinculación.

En cuanto a la situación deseada de la Dimensión Transformación /Explotación (Gráfico 3), el enfoque por parte de las empresas y academia hacia la problemática a solucionar o mejorar es calificado con nivel medio por parte de la percepción del responsable de la empresa. Invita a poner una mayor atención al nivel de compromiso de todas las partes involucradas. Los beneficios obtenidos por la vinculación son calificados en nivel medio contra la situación deseada.El estudiante cumple en casi su totalidad con el proyecto a ejecutar; es deficiencia de la empresa en cuanto a recursos económicos,personal para el seguimiento o implementación del proyecto resultado de la vinculación academia-industria, desaprovechando en un $50 \%$ la transformación y explotación del conocimiento de la fuente externa.

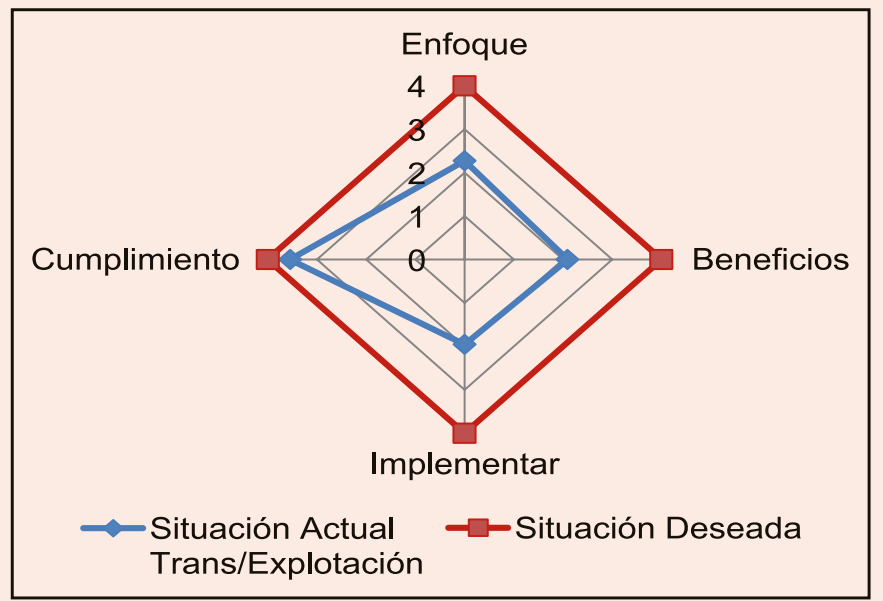

Gráfico 3. Situación Actual vs Deseada Transformación/ Explotación.

\section{DISCUSIÓN}

Los resultados obtenidos señalan el cumplimiento del objetivo principal de este estudio: conocer la percepción de los empresarios hacia los proyectos de vinculación. Los participantes percibieron como "buena" la cooperación obtenida por parte del Posgrado, con apertura para participar con estudiantes de próximas generaciones. Una de las interrogantes que se aclaran en esta investigación, es el cómo se adquiere el conocimiento por parte de la empresa [9]. Destaca el hecho de que el $90 \%$ de los proyectos de vinculación se dieron gracias al acercamiento de los estudiantes, a diferencia de otros estudios que establecen que la vinculación proviene por la relación de los maestros en la industria [10]. Desarrollar la capacidad de comunicación y trabajo en equipo por parte de las empresas es primordial para aplicar y asimilar los conocimientos de las fuentes externas exitosamente [11]. En cuanto a la asimilación del conocimiento, el $80 \%$ 
de los estudiantes captó en su totalidad las problemáticas presentadas por las empresas. En lo que respecta a la implementación y transformación, el $85 \%$ de las empresas presentó un impacto positivo, pero en un poco más de la mitad no se llevó a cabo la explotación del conocimiento debido a la falta del recurso humano para la implementación de las mejoras. Estos hallazgos permitirán proponer estrategias que pueden ser replicadas para mejorar los mecanismos y procedimientos de vinculación aportando a los estudiantes una formación integral para transmitir conocimientos; y a las empresas la facilidad para adquirir, asimilar y explotar estos conocimientos incidiendo siempre en el bienestar y desarrollo social.

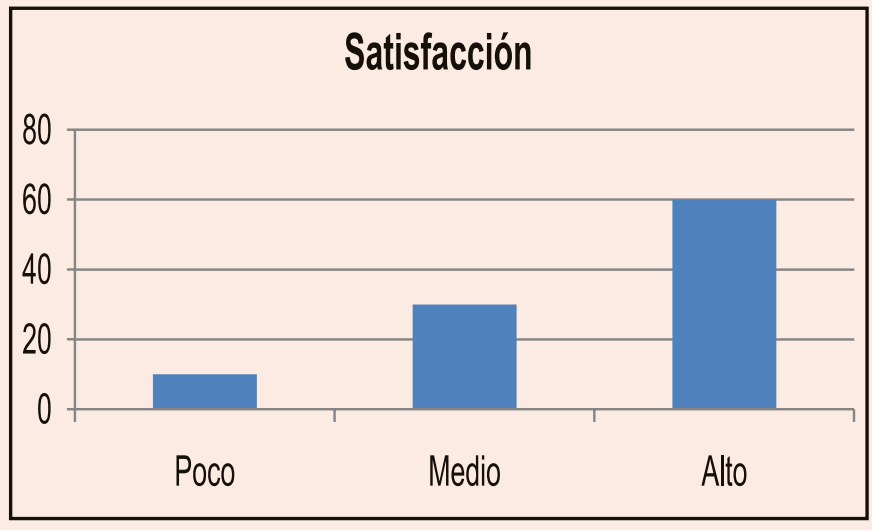

Gráfico 4. Nivel de Satisfacción Vinculación.

\section{CONCLUSIONES}

Una vez que se ha definido la situación actual en cuanto a cada una de las dimensiones de la capacidad de absorción del conocimiento en las empresas que se han vinculado con el Posgrado en Ingeniería Industrial, se concluye que la Adquisición del Conocimiento por parte de las empresas es muy bajo en su relación con la academia. La empresa no tiene la cultura de acercamiento, ya que en el $90 \%$ de los casos de colaboración fue iniciativa del estudiante promover la vinculación. Las empresas están desaprovechando esta valiosa fuente externa de conocimiento (gratuita). La falta de personal encargado de percatarse de fuentes externas de conocimiento disminuye la posibilidad de innovar. En cuanto a la Dimensión Asimilación, el empresario percibe la falta de respaldo por parte del Director de Tesis al Estudiante; así como la imagen ante la empresa. No obstante, las necesidades de la empresa han sido captadas casi en su totalidad. El involucramiento por partes de todas las partes: Empresarios, Directores de tesis y Estudiantes enriquecerá la forma en que la problemática es asimilada y facilitará la transformación e implementación del proyecto. Una vez que el conocimiento es adquirido no representa ninguna dificultad por parte del estudiante adaptar el conocimiento y asimilar las necesidades de la empresa. En la Dimensión Transformación y Explotación el alumno cumple completamente con los objetivos propuestos por las empresas, solución o mejora de problemáticas; es en la parte de la implementación explotación de los conocimientos que las empresas necesitan reforzar; el $50 \%$ de los casos no implementa el conocimiento obtenido debido a falta de seguimiento o recursos. En esta parte final se entrelaza la Dimensión de Asimilación; donde la empresa deberá reforzar su visión en el involucramiento de personal necesario; ya que una vez concluido el proyecto se pueda implementar el resultado obtenido agregando valor a la organización.

\section{BIBLIOGRAFÍA}

[1] W.M. Cohen and Da Levinthal. "Absorptive capacity; a new perspective of learning and innovation". Administrative Science Quarterly, Vol. 35, No.1,1990, pp 128-152.

[2] G. Todorova and G. Durisin. "Absorptive capacity: Valuing a reconceptualization". Academy of Management Review, Vol.32, No 3, 2007, pp. 774-786.

[3] P.J. Lane, B.R. Koka, and S. Pathak. "The reification of absorptive capacity: A critical review and rejuvenation of the construct". Academy of Management Review, Vol. 31, 2006, No. 4, pp 833-63.

[4] P.J. Lane and M. Lubatkin. "Relative absorptive capacity and interorganizational learning". Strategic Management Journal, Vol.19,1998, pp 111-125.

[5] S. A. Zhara and G. George. "Absorptive Capacity: A Review, Reconceptualization, and Extension". Academy of Management Review, Vol. 27, No.2, 2002, pp 185- 203.

[6] G. Szulanski, "Exploring internal stickiness: Impediments to the transfer of best practice within the firm". Strategic Management Journal, Vol. No. 17, 1996, pp 27-43.

[7] G.S. Van der Vegt and O. Janssen. "Joint impact of interdependence and group diversity on innovation". Journal of Management, Vol.29, No. 5, 2003, pp 729-51.

[8] D. George and P. Mallery, "SPSS for Windows step by step: A simple guide and reference, 11.0 update", (4th ed.), 2003, Boston: Allyn \& Bacon.

[9] C. Enrique, S. Cárdenas, D. Arellano y E. Ramírez, "La vinculación entre la universidad y la industria en México. Una revisión a los hallazgos de la Encuesta Nacional de Vinculación", Perfiles educativos, Vol. 33, 2011, pp. 187-199.

[10] M. Coronado y A. Tapia, "Vinculación universidad-sector productivo: un estudio de la industria alimentaria", Comercio Exterior, Banco Nacional de Comercio Exterior, Vol. 46, No. 10, 1996, pp. 1-9.

[11] P. Mario, "Estudio de Vinculación Empresas-IES", Alianza FIIDEM, A.C., Vol. 1, 2013, pp. 8-9.

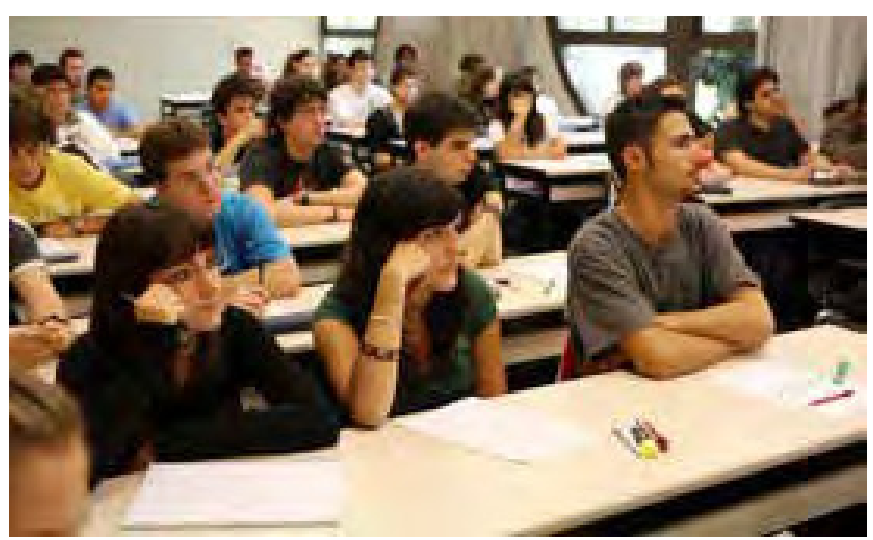

